\title{
Discovery of extended VHE $\gamma$-ray emission from the vicinity of the young massive stellar cluster Westerlund 1
}

H.E.S.S. Collaboration: A. Abramowski ${ }^{1}$, F. Acero ${ }^{2}$, F. Aharonian ${ }^{3,4,5}$, A. G. Akhperjanian ${ }^{6,5}$, G. Anton ${ }^{7}$, A. Balzer ${ }^{7}$, A. Barnacka ${ }^{8,9}$, U. Barres de Almeida ${ }^{10, \star}$, Y. Becherini ${ }^{11,12}$, J. Becker ${ }^{13}$, B. Behera ${ }^{14}$, K. Bernlöhr ${ }^{3,15}$, E. Birsin ${ }^{15}$, J. Biteau ${ }^{12}$, A. Bochow ${ }^{3}$, C. Boisson ${ }^{16}$, J. Bolmont ${ }^{17}$, P. Bordas ${ }^{18}$, J. Brucker ${ }^{7}$, F. Brun ${ }^{12}$, P. Brun ${ }^{9}$, T. Bulik ${ }^{19}$, I. Büsching ${ }^{20}, 13$, S. Carrigan ${ }^{3}$, S. Casanova ${ }^{13}$, M. Cerruti ${ }^{16}$, P. M. Chadwick ${ }^{10}$, A. Charbonnier ${ }^{17}$, R. C. G. Chaves ${ }^{3}$, A. Cheesebrough ${ }^{10}$, L.-M. Chounet ${ }^{12}$, A. C. Clapson ${ }^{3}$, G. Coignet $^{21}$, G. Cologna ${ }^{14}$, J. Conrad ${ }^{22}$, M. Dalton ${ }^{15}$, M. K. Daniel ${ }^{10}$, I. D. Davids ${ }^{23}$, B. Degrange ${ }^{12}$, C. Deil ${ }^{3}$, H. J. Dickinson ${ }^{22}$, A. Djannati-Atai ${ }^{11}$, W. Domainko ${ }^{3}$, L. O'C. Drury ${ }^{4}$, F. Dubois ${ }^{21}$, G. Dubus ${ }^{24}$, K. Dutson ${ }^{25}$, J. Dyks ${ }^{8}$, M. Dyrda ${ }^{26}$, K. Egberts ${ }^{27}$, P. Eger ${ }^{7}$, P. Espigat ${ }^{11}$, L. Fallon ${ }^{4}$, C. Farnier ${ }^{2}$, S. Fegan ${ }^{12}$, F. Feinstein ${ }^{2}$, M. V. Fernandes ${ }^{1}$, A. Fiasson ${ }^{21}$, G. Fontaine ${ }^{12}$, A. Förster ${ }^{3}$, M. Füßling ${ }^{15}$, Y. A. Gallant ${ }^{2}$, H. Gast ${ }^{3}$, L. Gérard ${ }^{11}$, D. Gerbig ${ }^{13}$, B. Giebels ${ }^{12}$, J. F. Glicenstein ${ }^{9}$, B. Glück ${ }^{7}$, P. Goret ${ }^{9}$, D. Göring ${ }^{7}$, S. Häffner ${ }^{7}$, J. D. Hague ${ }^{3}$, D. Hampf ${ }^{1}$, M. Hauser ${ }^{14}$, S. Heinz ${ }^{7}$, G. Heinzelmann ${ }^{1}$, G. Henri ${ }^{24}$, G. Hermann ${ }^{3}$, J. A. Hinton ${ }^{25}$, A. Hoffmann ${ }^{18}$, W. Hofmann ${ }^{3}$, P. Hofverberg ${ }^{3}$, M. Holler ${ }^{7}$, D. Horns ${ }^{1}$, A. Jacholkowska ${ }^{17}$, O. C. de Jager ${ }^{20}$, C. Jahn ${ }^{7}$, M. Jamrozy ${ }^{28}$, I. Jung ${ }^{7}$, M. A. Kastendieck ${ }^{1}$, K. Katarzyński ${ }^{29}$, U. Katz ${ }^{7}$, S. Kaufmann ${ }^{14}$, D. Keogh ${ }^{10}$, D. Khangulyan ${ }^{3}$, B. Khélifi ${ }^{12}$, D. Klochkov ${ }^{18}$, W. Kluźniak ${ }^{8}$, T. Kneiske ${ }^{1}$, Nu. Komin ${ }^{21}$, K. Kosack ${ }^{9}$, R. Kossakowski ${ }^{21}$, H. Laffon ${ }^{12}$, G. Lamanna ${ }^{21}$, D. Lennarz ${ }^{3}$, T. Lohse ${ }^{15}$, A. Lopatin ${ }^{\text {, }}$ C.-C. $\mathrm{Lu}^{3}$, V. Marandon ${ }^{11}$, A. Marcowith ${ }^{2}$, J. Masbou ${ }^{21}$, D. Maurin ${ }^{17}$, N. Maxted ${ }^{30}$, M. Mayer ${ }^{7}$, T. J. L. McComb ${ }^{10}$, M. C. Medina ${ }^{9}$, J. Méhault ${ }^{2}$, R. Moderski ${ }^{8}$, E. Moulin ${ }^{9}$, C. L. Naumann ${ }^{17}$, M. Naumann-Godo ${ }^{9}$, M. de Naurois ${ }^{12}$, D. Nedbal ${ }^{31}$, D. Nekrassov ${ }^{3}$, N. Nguyen ${ }^{1}$, B. Nicholas ${ }^{30}$, J. Niemiec $^{26}$, S. J. Nolan ${ }^{10}$, S. Ohm ${ }^{32,25,3}$,

E. de Oña Wilhelmi ${ }^{3}$, B. Opitz ${ }^{1}$, M. Ostrowski ${ }^{28}$, I. Oya ${ }^{15}$, M. Panter ${ }^{3}$, M. Paz Arribas ${ }^{15}$, G. Pedaletti ${ }^{14}$, G. Pelletier ${ }^{24}$, P.-O. Petrucci ${ }^{24}$, S. Pita ${ }^{11}$, G. Pühlhofer ${ }^{18}$, M. Punch ${ }^{11}$, A. Quirrenbach ${ }^{14}$, M. Raue ${ }^{1}$, S. M. Rayner ${ }^{10}$, A. Reimer ${ }^{27}$,

O. Reimer ${ }^{27}$, M. Renaud ${ }^{2}$, R. de los Reyes ${ }^{3}$, F. Rieger ${ }^{3,33}$, J. Ripken ${ }^{22}$, L. Rob ${ }^{31}$, S. Rosier-Lees ${ }^{21}$, G. Rowell ${ }^{30}$, B. Rudak $^{8}$, C. B. Rulten ${ }^{10}$, J. Ruppel ${ }^{13}$, V. Sahakian ${ }^{6,5}$, D. Sanchez ${ }^{3}$, A. Santangelo ${ }^{18}$, R. Schlickeiser ${ }^{13}$, F. M. Schöck ${ }^{7}$, A. Schulz ${ }^{7}$, U. Schwanke ${ }^{15}$, S. Schwarzburg ${ }^{18}$, S. Schwemmer ${ }^{14}$, F. Sheidaei ${ }^{11,20}$, M. Sikora ${ }^{8}$, J. L. Skilton ${ }^{3}$, H. Sol ${ }^{16}$, G. Spengler ${ }^{15}$, Ł. Stawarz ${ }^{28}$, R. Steenkamp ${ }^{23}$, C. Stegmann ${ }^{7}$, F. Stinzing ${ }^{7}$, K. Stycz ${ }^{7}$, I. Sushch ${ }^{15, \star \star}$, A. Szostek ${ }^{28}$, J.-P. Tavernet ${ }^{17}$, R. Terrier ${ }^{11}$, M. Tluczykont ${ }^{1}$, K. Valerius ${ }^{7}$, C. van Eldik ${ }^{3}$, G. Vasileiadis ${ }^{2}$, C. Venter ${ }^{20}$, J. P. Vialle ${ }^{21}$, A. Viana ${ }^{9}$, P. Vincent ${ }^{17}$, H. J. Völk ${ }^{3}$, F. Volpe ${ }^{3}$, S. Vorobiov ${ }^{2}$, M. Vorster ${ }^{20}$, S. J. Wagner ${ }^{14}$, M. Ward ${ }^{10}$, R. White ${ }^{25}$, A. Wierzcholska ${ }^{28}$, M. Zacharias ${ }^{13}$, A. Zajczyk ${ }^{8,2}$, A. A. Zdziarski ${ }^{8}$, A. Zech ${ }^{16}$, and H.-S. Zechlin ${ }^{1}$

(Affiliations can be found after the references)

Received 22 August 2011 / Accepted 12 October 2011

\section{ABSTRACT}

\begin{abstract}
Aims. Results obtained in very-high-energy (VHE; $E \geq 100 \mathrm{GeV}$ ) $\gamma$-ray observations performed with the H.E.S.S. telescope array are used to investigate particle acceleration processes in the vicinity of the young massive stellar cluster Westerlund 1 (Wd 1).

Methods. Imaging of Cherenkov light from $\gamma$-ray induced particle cascades in the Earth's atmosphere is used to search for VHE $\gamma$ rays from the region around Wd 1. Possible catalogued counterparts are searched for and discussed in terms of morphology and energetics of the H.E.S.S. source. Results. The detection of the degree-scale extended VHE $\gamma$-ray source HESS J1646-458 is reported based on 45 h of H.E.S.S. observations performed between 2004 and 2008. The VHE $\gamma$-ray source is centred on the nominal position of Wd 1 and detected with a total statistical significance of $\sim 20 \sigma$. The emission region clearly extends beyond the H.E.S.S. point-spread function (PSF). The differential energy spectrum follows a power law in energy with an index of $\Gamma=2.19 \pm 0.08_{\text {stat }} \pm 0.20_{\text {sys }}$ and a flux normalisation at $1 \mathrm{TeV}$ of $\Phi_{0}=\left(9.0 \pm 1.4_{\text {stat }} \pm 1.8_{\text {sys }}\right) \times 10^{-12} \mathrm{TeV}^{-1} \mathrm{~cm}^{-2} \mathrm{~s}^{-1}$. The integral flux above $0.2 \mathrm{TeV}$ amounts to $(5.2 \pm 0.9) \times 10^{-11} \mathrm{~cm}^{-2} \mathrm{~s}^{-1}$.

Conclusions. Four objects coincident with HESS J1646-458 are discussed in the search of a counterpart, namely the magnetar CXOU J164710.2-455216, the X-ray binary 4U 1642-45, the pulsar PSR J1648-4611 and the massive stellar cluster Wd 1. In a single-source scenario, Wd 1 is favoured as site of VHE particle acceleration. Here, a hadronic parent population would be accelerated within the stellar cluster. Beside this, there is evidence for a multi-source origin, where a scenario involving PSR J1648-4611 could be viable to explain parts of the VHE $\gamma$-ray emission of HESS J1646-458.
\end{abstract}

Key words. open clusters and associations: general - gamma rays: stars - astroparticle physics

\footnotetext{
* Supported by CAPES Foundation, Ministry of Education of Brazil.

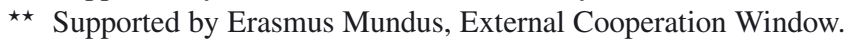




\section{Introduction}

The long-standing question on the origin and acceleration mechanisms of hadronic and leptonic Galactic cosmic rays (GCRs) is still not settled, despite considerable progress. The detection of very-high-energy (VHE) $\gamma$-ray emission from shell-type supernova remnants (SNRs), e.g. Cassiopeia A, RX J1713-3946, RX J0852.0-4622, RCW 86, SN 1006 (summarised in Hinton \& Hofmann 2009), and recently HESS J1731-347 (H.E.S.S. Collaboration et al. 2011) and Tycho's SNR (Acciari et al. 2011) supports the widely accepted idea of SNRs being acceleration sites of GCRs. It has been noted for many years that the Galactic SNR population provides sufficient energy input to sustain the CR flux measured at Earth. The underlying theory assumes that electrons and protons are injected into SNR shock fronts where they are accelerated via the diffusive shock acceleration process up to energies of $\sim 10^{15} \mathrm{eV}$ (Krymskii 1977; Axford et al. 1977; Bell 1978; Blandford \& Ostriker 1978). The ability of SNRs to accelerate electrons up to the so-called knee in the differential energy spectrum of the GCRs and our common belief that this holds for protons, too, constitute the paradigm that SNRs are the long-thought sources of GCRs. In interactions with the ambient medium, i.e. matter and electromagnetic fields, these GCRs then produce VHE $\gamma$ rays which can be detected by current imaging atmospheric Cherenkov telescope (IACT) systems, e.g. H.E.S.S., MAGIC, VERITAS or CANGAROO-III. Additionally, evolving SNRs could explain the chemical composition up to the knee region. Furthermore, core-collapse supernovae could explain observed overabundances of some isotopes, e.g. ${ }^{22} \mathrm{Ne}$ (Higdon \& Lingenfelter 2003). However, recent studies applied to RX J1713-3946 highlight potential problems for a dominant hadronic interpretation for this object (Ellison et al. 2010) and motivate the search for other acceleration sites and processes.

SNR shells are not the only sites in the Galaxy where GCRs can be produced via diffusive shock acceleration. One alternative scenario is particle acceleration in strong shocks in colliding wind binaries (CWBs). Massive stars are to a large extent bound in binary systems (e.g. Zinnecker 2003; Gies 2008), generally exhibit high mass-loss rates $\left(10^{-5} M_{\odot} \mathrm{yr}^{-1}-10^{-3} M_{\odot} \mathrm{yr}^{-1}\right)$ and drive strong supersonic winds with velocities of the order of a few $10^{3} \mathrm{~km} \mathrm{~s}^{-1}$. When these winds collide in a stellar binary system they form a wind-wind interaction zone where charged particles can be accelerated to high energies (e.g. Eichler \& Usov 1993). Electrons can then up-scatter stellar photons present in the wind collision zones via the inverse Compton (IC) process to GeV energies (Mücke \& Pohl 2002; Manolakou et al. 2007). On the other hand, relativistic nucleons can inelastically scatter with particles in the dense wind and produce $\pi^{0} \mathrm{~s}$ which subsequently decay into VHE $\gamma$ rays (Benaglia \& Romero 2003; Bednarek 2005; Domingo-Santamaría \& Torres 2006; Reimer et al. 2006). Apart from acceleration in binaries, GCRs can be accelerated in the winds of single massive stars (e.g. Montmerle 1979).

Another scenario involves collective stellar winds: It is commonly accepted that the bulk (if not all) of the corecollapse SN progenitor stars and CWBs evolve from collapsing gas condensations in giant molecular clouds (e.g. Zinnecker $\&$ Yorke 2007) and mostly remain close to their birthplaces in groups of loosely bound associations or dense stellar clusters. When the winds of multiple massive stars in such systems collide they form a collective cluster wind which drives a giant bubble $(O(100 \mathrm{pc}))$, also referred to as superbubble (SB), filled with a hot $\left(T \approx 10^{6} \mathrm{~K}\right)$ and tenuous $\left(n<1 \mathrm{~cm}^{-3}\right)$ plasma (e.g.
Weaver et al. 1977; Silich et al. 2005). At the wind interaction zones, e.g. at the termination shock of the stellar cluster wind, turbulences in form of magneto-hydrodynamic (MHD) fluctuations and weak reflected shocks can build up. Unlike SNR shock fronts and CWBs where GCRs are accelerated through the 1st order Fermi acceleration, turbulences in SB interiors can accelerate particles to very high energies also via the 2 nd order Fermi mechanism (e.g. Bykov 2001). Moreover, after a few million years, supernova explosions of massive stars $\left(M>8 M_{\odot}\right)$ in the thin and hot SB environment eventually lead to efficient particle acceleration at the boundary of the SB or at MHD turbulences and further amplify existing MHD turbulences (e.g. Ferrand \& Marcowith 2010, and references therein). The interaction of these GCRs with the ambient medium including molecular clouds or electromagnetic fields leads to the production of VHE $\gamma$ rays which can then be studied on Earth. Therefore, stellar clusters are promising targets to study acceleration and propagation processes of GCRs.

One of the most prominent objects among stellar clusters in the Galaxy is Westerlund 1 (Wd 1). After its discovery in 1961 (Westerlund 1961) subsequent observations have established Wd 1 as the most massive stellar cluster in our Galaxy placing a lower limit on its mass of $10^{5} M_{\odot}$ (Clark et al. 2010). An unprecedented accumulation of evolved massive stars is found without indication of the presence of an early-type mainsequence star. Amongst the most massive stars, 24 Wolf-Rayet stars (binary fraction $\geq 62 \%$ ) have been detected and a number of $\sim 150$ OB super- and hypergiants (binary fraction $\sim 30 \%$ ) is expected (Crowther et al. 2006; Dougherty et al. 2010, and references therein).

The analysis of Chandra data revealed an arc minute-scale extended diffuse X-ray emission (Muno et al. 2006b) which is only seen for a few young stellar associations in the Galaxy, for example RCW 38 (Wolk et al. 2002) and possibly the Arches cluster (Law \& Yusef-Zadeh 2004) as well as in the Large Magellanic Cloud in 30 Doradus C (Bamba et al. 2004) and DEM L192 (Cooper et al. 2004). The total X-ray luminosity of the observed diffuse emission within $5^{\prime}$ of $\mathrm{Wd} 1$ is dominated by its non-thermal component and amounts to $L_{\mathrm{X}} \approx 3 \times 10^{34} \mathrm{erg} \mathrm{s}^{-1}$ which represents just a fraction of $10^{-5}$ of the total mechanical power in this system (Muno et al. 2006b). However, models as in Oskinova (2005) predict a thermal X-ray luminosity of $\sim 10^{37} \mathrm{erg} \mathrm{s}^{-1}$ for stellar clusters comparable to Wd 1, which was clearly not observed by Chandra for Wd 1 . As for previous observations, there remains the open question into which channel most of the unobserved energy is dissipated.

The detection of VHE $\gamma$-ray emission from HESS J1646-458 was initially reported in Ohm et al. (2010a) and Ohm et al. (2010b). This paper focuses on a detailed spectral and morphological study of the emission region and investigates a possible multi-source origin. An in-depth search for plausible counterparts is conducted and possible acceleration-mechanism scenarios are elaborated.

\section{H.E.S.S. observations and data analysis}

Given the large $\sim 5^{\circ}$ field of view (FoV) combined with the good off-axis sensitivity, observations with H.E.S.S. are perfectly suited to cover the vicinity of $\mathrm{Wd} 1$ and allow for the detailed morphological study of extended sources such as HESS J1646-458. Thereby any large-scale non-thermal VHE $\gamma$-ray emission around Wd 1 can be probed. 


\subsection{The H.E.S.S. experiment}

The High Energy Stereoscopic System (H.E.S.S.) is an array of four imaging atmospheric Cherenkov telescopes located in the Khomas Highland of Namibia, $1800 \mathrm{~m}$ above sea level. The telescopes are identical in construction and each one is comprised of a $107 \mathrm{~m}^{2}$ optical reflector composed of segmented spherical mirrors. These focus the incident light into a fine-grained camera built of 960 photomultiplier tubes. By means of the imaging atmospheric Cherenkov technique (see e.g. Hillas 1985) Cherenkov light, emitted by the highly-relativistic charged particles in extensive air showers, was imaged by the mirrors onto the Cherenkov camera. A single shower was recorded by multiple telescopes under different viewing angles. This allowed for the stereoscopic reconstruction of the primaries' direction and energy with an average energy resolution of $15 \%$ and an eventby-event angular resolution better than $0.1^{\circ}$ (Gaussian standard deviation, Aharonian et al. 2006a).

\subsection{The data set}

The region around Wd 1 was observed during the H.E.S.S. Galactic Plane Survey (GPS) in 2004 and 2007 (Aharonian et al. 2006c; Chaves et al. 2008). Additionally, follow-up observations pointing in the direction of $\mathrm{Wd} 1$ have been performed from May to August 2008. Data taken under unstable weather conditions or with malfunctioning hardware were excluded in the standard data quality selection procedure (Aharonian et al. 2006a). Also, pointed observations on Wd 1 at very large zenith angles of more than $55^{\circ}$ were excluded due to systematic effects in the description of the camera acceptance at such low altitudes for an extended source like HESS J1646-458. After quality selection and dead time correction the total observation time of $45.1 \mathrm{~h}$ was reduced to a live time of $33.8 \mathrm{~h}$. Observations have been carried out at zenith angles from $21^{\circ}$ to $45^{\circ}$ with a mean value of $26^{\circ}$ and an average pointing offset from the $\mathrm{Wd} 1$ position of $1.1^{\circ}$.

\subsection{Analysis technique}

The data set presented here was processed using the H.E.S.S. Standard Analysis for shower reconstruction (Aharonian et al. 2006a) and the Boosted Decision Trees (BDT) method to suppress the hadronic background component (Ohm et al. 2009) ${ }^{1}$. By parametrising the centre of gravity and second moments of the recorded extensive air shower image (Hillas 1985) in multiple telescopes the shower geometry of the incident primary particle was reconstructed stereoscopically. The directional information together with the measured image intensity was used to reconstruct the energy of the event. Since observations have been conducted over four years, the optical reflectivity of the H.E.S.S. mirrors varied and the gains of the photomultipliers changed. This effect has been taken into account in the spectral reconstruction by calibrating the energy of each event with single muon rings (Aharonian et al. 2006a). The decision tree-based machine learning algorithm BDT returns a continuous variable called $\zeta$ which was used to select $\gamma$-ray-like events. Cutting on this parameter results in an improvement in terms of sensitivity compared to the H.E.S.S. Standard Analysis of $\sim 20 \%$ and $\sim 10 \%$ for spectral and morphological analysis, respectively (Ohm et al. 2009).

\footnotetext{
1 The software which was used to analyse the VHE $\gamma$-ray data presented in this work is the H.E.S.S. analysis package (HAP) in version 10-06-pl07.
}

Similar to the H.E.S.S. Standard Analysis, two sets of $\gamma$-ray selection cuts have been defined in Ohm et al. (2009). For the production of sky images the $\zeta_{\text {hard }}$ - $c$ uts are used. They require a minimum intensity of 160 photo electrons (p.e.) in each camera image yielding a superior angular resolution of less than $0.1^{\circ}$ even at large offsets of $2.5^{\circ}$ from the telescope pointing position. Additionally, $30 \%$ more background events are rejected resulting in a $10 \%$ higher sensitivity compared to the H.E.S.S. Standard Analysis. For the spectral analysis a low energy threshold is desirable for a broad energy coverage and achieved by applying the $\zeta_{\text {std }}$-cuts with a 60 p.e. cut on the image intensity. For the data set under study, this infers an energy threshold of $450 \mathrm{GeV}$ for spectral analysis and $700 \mathrm{GeV}$ for morphological analysis.

For two-dimensional sky image generation and morphology studies, the template background model (Rowell 2003; Berge et al. 2007) is applied. For this method the CR background is estimated in parameter space rather than in angular space. In this analysis, the BDT output parameter $\zeta$ has been used to define signal and background regions. The normalisation $\alpha$ between signal and background is calculated as the fraction of all events in the FoV falling into the signal regime, excluding source regions, divided by the number of all events in the FoV in the background regime, again excluding all source regions. The system acceptance to measure $\gamma$-ray like and CR-like events drops off radially with the distance to the telescope pointing position. Since this acceptance is different for both types of events, a correction is applied to $\alpha$. Sky images obtained with the template background model agree with sky images generated with the ring background method. The ring background method estimates the signal-like hadronic $\mathrm{CR}$ contribution at each trial position on the sky by integrating events in an annulus centred on that position, excluding potential source regions.

Figure 1 shows the VHE $\gamma$-ray count map of the region around $\mathrm{Wd} 1$ and reveals very extended $\gamma$-ray emission. The complex morphology apparent in the sky image and a potential multi-source origin of the emission is investigated and discussed in detail in Sect. 3. Since it is not possible to estimate the background from the same FoV due to the fact that observations have been carried out within regions of VHE $\gamma$-ray emission, the $\mathrm{On}$ Off background estimation method is utilised to extract spectral information for the whole emission region, indicated as a white circle in Fig. 1. Here, the CR background is subtracted from the source region (On data) using extragalactic observations taken without any VHE $\gamma$-ray signal in the FoV (Off data). To ensure similar observational conditions for $O n$ and $O f f$ data, only OnOff pairs of observations are considered that were taken at similar zenith angles and within four months of each other, resulting in a total live time of $20 \mathrm{~h}$ for the On data set used for spectral analysis. The absolute normalisation $\alpha$ between $O n$ and Off data is calculated using the fraction of total events in both observations (again, excluding potential source regions). The reflected background method (Berge et al. 2007) is used to derive spectral information for smaller regions and the full data set as discussed in Sects. 3.2 and 3.3.

All studies presented in this work were cross-checked by a second analysis chain which is based on the H.E.S.S. standard event reconstruction scheme (Aharonian et al. 2006a) using the Hillas second moment method (Hillas 1985) and an independent calibration of pixel amplitudes and identification of problematic or dead pixels in the IACT cameras. Additionally, the Model Analysis (de Naurois \& Rolland 2009) for the selection of $\gamma$-ray-like events has been utilised to cross-check the spectral results. All analyses give compatible results. 


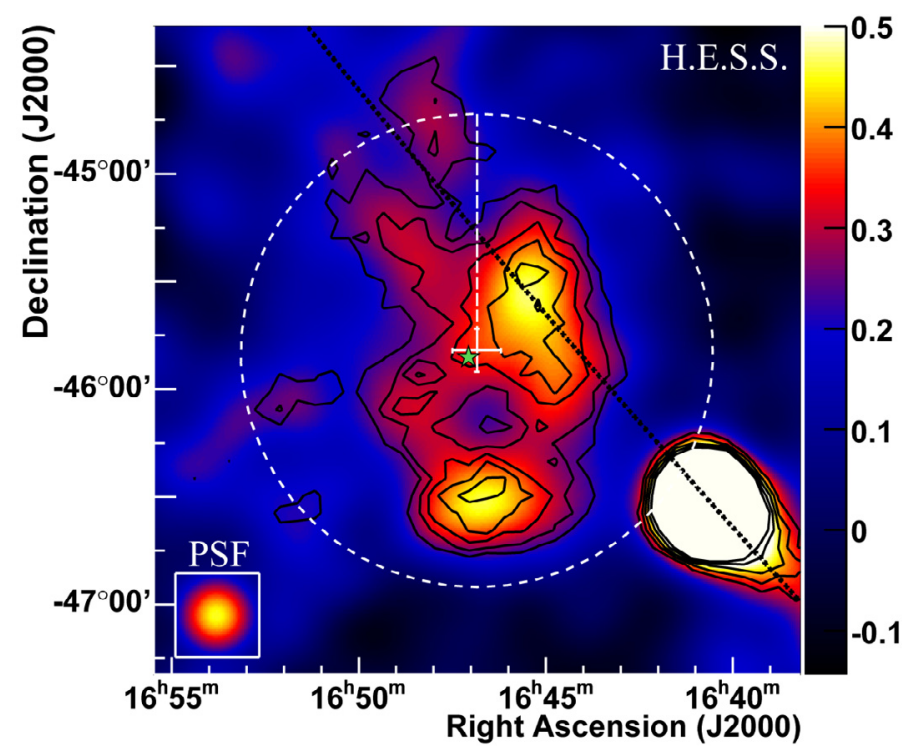

Fig. 1. H.E.S.S. excess map of the region around Wd 1 corrected for the camera acceptance, in units of equivalent on-axis VHE $\gamma$-ray events per $\operatorname{arcmin}^{2}$ and obtained with the template background method. The image is smoothed with a 2D Gaussian kernel with a variance of $0.12^{\circ}$ to reduce the effect of statistical fluctuations. Significance contours between $4 \sigma$ and $8 \sigma$ are overlaid in black, obtained by integrating events within a radius of 0.22 degrees at each given position. The green star marks the position of Wd 1, the white cross the best fit position of the VHE $\gamma$-ray emission and the black dashed line the Galactic plane. The inlay in the lower left corner represents the size of a point-like source as it would have been seen by H.E.S.S. for this analysis and the same smoothing, normalised to the maximum of HESS J1646-458. The dotted white circle has a radius of $1.1^{\circ}$ and denotes the region which was used for the spectral reconstruction of the VHE $\gamma$-ray emission. Note that the bright region in the lower right corner is the source HESS J1640-465 detected during the GPS (Aharonian et al. 2006c).

\section{VHE results}

\subsection{Position}

Figure 1 shows a background-subtracted, camera acceptancecorrected image of the VHE $\gamma$-ray counts per $\operatorname{arcmin}^{2}$ of the $3^{\circ} \times 3^{\circ} \mathrm{FoV}$ centred on the best fit position of the $\gamma$-ray excess as obtained with the template background method. The acceptance correction has been performed using $\gamma$-ray like background events that pass the $\gamma$-ray selection cuts. The map is smoothed with a Gaussian kernel with a variance of $0.12^{\circ}$ to reduce the effect of statistical fluctuations and to highlight significant morphological features. Significance contours from $4 \sigma$ to $8 \sigma$ are overlaid after integrating events within a radius of $0.22^{\circ}$ at each trial source position. This integration radius is matched to the rms of the Gaussian to resample significant features in the sky image and is chosen a priori to match the integration radius typically used in the GPS analysis for the search of slightly extended sources (Aharonian et al. 2006c). Given the extended and complex morphology of the VHE $\gamma$-ray emission the position obtained from a two-dimensional Gaussian fit convolved with the H.E.S.S. PSF to the raw excess count map obtained for $\zeta_{\text {hard }}$-cuts is used to derive an estimate on the centre of gravity of the emission. The two-dimensional Gaussian fit gives a best fit position of RA $16^{\mathrm{h}} 46^{\mathrm{m}} 50^{\mathrm{s}} \pm 27^{\mathrm{s}}$ and Dec $-45^{\circ} 49^{\prime} 12^{\prime \prime} \pm 7^{\prime}$ (J2000). Within statistical errors the centre of gravity of the VHE $\gamma$-ray emission is consistent with the nominal Wd 1 cluster position of RA $16^{\mathrm{h}} 47^{\mathrm{m}} 00.40^{\mathrm{s}}$ and Dec $-45^{\circ} 51^{\prime} 04.9^{\prime \prime}$ (J2000). Based

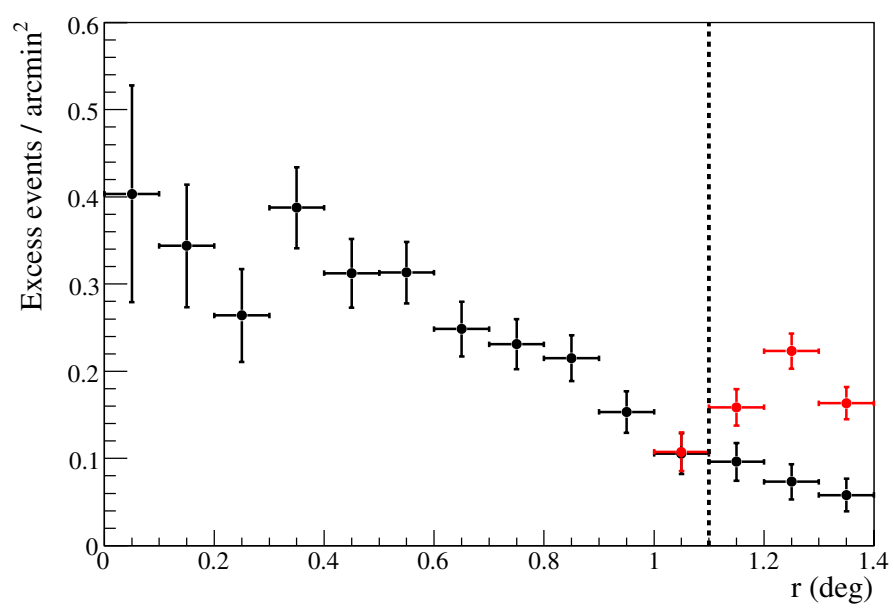

Fig. 2. H.E.S.S. radial profile relative to the best-fit position of the VHE $\gamma$-ray emission. The dotted vertical line denotes the $95 \%$ containment radius used to obtain the spectrum shown in Figure 4. Note that the region covering the bright source HESS J1640-465 (see Figure 1) has been excluded for the radial profile shown in black by omitting a circle segment with $220^{\circ} \leq \phi \leq 260^{\circ}$ for radii of $1.0^{\circ} \leq r \leq 1.4^{\circ}$. The red graph displays the radial profile without excluding HESS J1640-465.

on the radial profile shown in Fig. 2 the $95 \%$ containment radius of the VHE $\gamma$-ray emission relative to the best fit position is determined to be $1.1^{\circ}$. This radius is used to extract the energy spectrum presented in Sect. 3.3. Note that although the sky image gives the impression that the region used for spectral reconstruction is contaminated by $\gamma$ rays from HESS J1640-465, this is mostly an artifact of the smoothing procedure. The real contribution is less than $10 \%$ in a ring between 1.0 and 1.1 degree from the best fit position and only $0.8 \%$ in the whole spectral extraction region. Within the integration region of $1.1^{\circ}$ a total of $2771 \pm 139 \gamma$-ray excess events at a significance level of $20.9 \sigma$ pre-trials $(20.1 \sigma$ post-trials $)$ are found.

\subsection{Morphology}

In order to investigate the multi-source hypothesis two emission regions $A$ and $B$ (shown in Fig. 3 (left)) are considered. The radii of $0.35^{\circ}$ and $0.25^{\circ}$ of region $A$ and $B$, respectively, are chosen according to the widths of the two substructures. A one-dimensional slice in the uncorrelated excess image along the major axis between the two regions has been produced. The fit of two separate sources with Gaussian shape results in a $\chi^{2}$ of 2.0 for 4 degrees of freedom with a probability of $74 \%$. The probability that the emission is explained by a single Gaussian profile or a constant value is found to be rather low at $0.2 \%$ and $0.1 \%$, respectively. An $F$-test also supports the multi-source hypothesis, given that the probabilities that the constant or single Gaussian emission models are preferred over the double Gaussian fit are $<0.02$ and $<0.01$, respectively.

Figure 3 (left) also suggests a contribution from diffuse VHE $\gamma$-ray emission along the Galactic plane which extends $1^{\circ}$ to $2^{\circ}$ from region $A$ north-eastwards. This impression is supported by the one-dimensional slice shown in Fig. 3 (bottom right), where the significance of the emission in all bins with distance $0.5^{\circ} \leq d \leq 1.8^{\circ}$ from the centre of region $A$ is between $2 \sigma$ and $4 \sigma$. This diffuse emission could be due to unresolved VHE $\gamma$-ray sources or a Galactic diffuse emission component, caused by the interaction of GCRs with molecular 

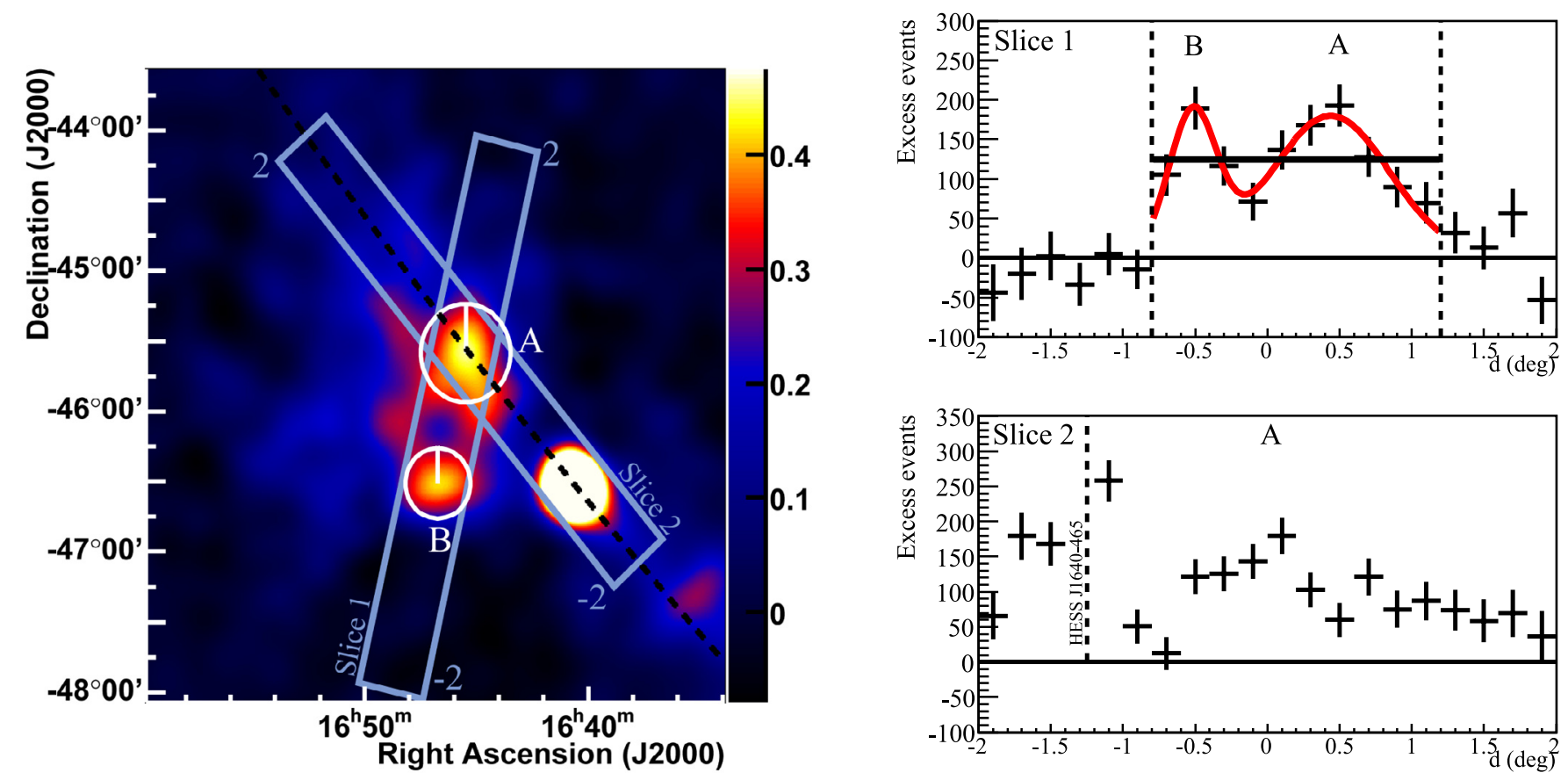

Fig. 3. Left: H.E.S.S. excess map as shown in Fig. 1 but for an enlarged region of $4.5^{\circ} \times 4.5^{\circ}$. The blue-grey boxes denote the regions used to generate the one-dimensional slices shown on the right. The white circles denote regions $A$ and $B$ which were used for spectral reconstruction (Table 2). The weak $\gamma$-ray emission seen in the lower right corner next to HESS J1640-465 is HESS J1634-472, also detected during the GPS (Aharonian et al. 2006c). Right, top: distribution of VHE $\gamma$-ray excess events in the blue-grey box, oriented along the two emission regions associated to HESS J1646-458 and starting at low Declination angles. The results of a fit of a constant and of two sources with Gaussian shape are indicated as black and red line, respectively. Right, bottom: same as top, but oriented along the Galactic plane, starting at low Right Ascension angles, close to the bright source HESS J1640-465 at $d=-1.25^{\circ}$. The slice has been truncated at 350 excess events in order to emphasize the VHE $\gamma$-ray emission from HESS J1646-458.

material located along the Galactic plane. As will be shown later in Fig. 6, there is indeed molecular material located in this region which could act as target for the interaction with CRs (as described in e.g. Casanova et al. 2009) and could account for part of the observed emission. The statistics of region $A$ and $B$ compared to the entire source region as obtained with the template background method are given in Table 1.

The studies presented here show some evidence for a multisource morphology and a separation into multiple VHE $\gamma$-ray sources. Moreover, spectral variations across the whole emission region, e.g. as observed for HESS J1825-137 (Aharonian et al. 2006d) or in the case of the H.E.S.S. sources in the Westerlund 2 field (Abramowski et al. 2011), could be apparent, which would further support the multi-source hypothesis. In this case, an energy-dependent morphology can be expected. To test this hypothesis, the complete data set has been divided into a low-energy band, containing events with reconstructed energies $E<1.0 \mathrm{TeV}$ and a high-energy band, containing events with reconstructed energies $E>1.0 \mathrm{TeV}$. The unsmoothed excess maps in coarse bins of $0.3^{\circ}$ width are used to test the underlying distribution. A $\chi^{2}$ test is performed using the number of excess events in each bin and reveals a value of 95.4 for 76 degrees of freedom (four bins covering HESS J1640-465 have been excluded in the calculation). Prior to the test a $\chi^{2}$ probability $p_{0}$ of 0.05 is defined to accept/reject the null hypothesis. The $p$-value of the test is $6.5 \%>p_{0}$, such that the null hypothesis that both excess maps follow the same underlying distribution cannot be rejected. Although no energy-dependent morphology can be detected from this test, a multi-source origin is preferred given the low probabilities for the single source fits.

\subsection{Spectrum}

The spectrum obtained for the whole emission region using the On-Off background estimation method is shown in Fig. 4. In the fit range between $0.45 \mathrm{TeV}$ and $75 \mathrm{TeV}$, the spectrum is well described by a power law: $\mathrm{d} N / \mathrm{d} E=\Phi_{0} \times$ $(E / 1 \mathrm{TeV})^{-\Gamma}$ with a photon index $\Gamma=2.19 \pm 0.08_{\text {stat }} \pm$ $0.20_{\text {sys }}$, and a differential flux normalisation at $1 \mathrm{TeV}$ of $\Phi_{0}$ $=\left(9.0 \pm 1.4_{\text {stat }} \pm 1.8_{\text {sys }}\right) \times 10^{-12} \mathrm{TeV}^{-1} \mathrm{~cm}^{-2} \mathrm{~s}^{-1}$. This translates into an integral flux above $0.2 \mathrm{TeV}$ of $F(>0.2 \mathrm{TeV})=$ $(5.2 \pm 0.9) \times 10^{-11} \mathrm{~cm}^{-2} \mathrm{~s}^{-1}$. The $\chi^{2}$ for the power law fit is 9.9 for 7 degrees of freedom, yielding a $\chi^{2}$ probability of $19 \%$.

Additionally, the differential energy spectra for region $A$ and $B$ are determined using the reflected background estimation method with results found to be consistent with the On-Off background technique. The integral flux above $0.2 \mathrm{TeV}$ as well as the spectral results obtained from a power-law fit are summarised in Table 2 and compared to the results for the spectral analysis of the whole $1.1^{\circ}$ region. The differential energy spectra for these two regions are shown in Fig. 4 as well. Within statistical errors, no change in photon index between the three studied regions is apparent, further supporting the lack of energy-dependent morphology across the source based on the current data.

\section{Discussion}

In this section, the spectral and morphological results are used to elaborate possible acceleration scenarios related to HESS J1646-458. Although the morphological analysis prefers a two-source approach (at the $\sim 2.5 \sigma$ level), the similarity of the 
Table 1. VHE $\gamma$-ray statistics for the regions shown in Figs. 1 and 3.

\begin{tabular}{lcccccccc}
\hline \hline Region & $\begin{array}{c}\text { RA (J2000) } \\
\text { deg }\end{array}$ & $\begin{array}{c}\text { Dec (J2000) } \\
\text { deg }\end{array}$ & $\begin{array}{c}\theta \\
\text { deg }\end{array}$ & $\begin{array}{c}\text { On } \\
\text { events }\end{array}$ & $\begin{array}{c}\text { Off } \\
\text { events }\end{array}$ & $\alpha$ & $\begin{array}{c}\text { Excess } \\
\text { events }\end{array}$ & $\begin{array}{c}\text { Significance } \\
\sigma\end{array}$ \\
\hline Full & 251.856 & -45.909 & 1.1 & 19032 & 1107471 & 0.014682 & 2771 & 20.1 \\
$A$ & 251.370 & -45.585 & 0.35 & 2313 & 120104 & 0.014998 & 511 & 10.0 \\
$B$ & 251.682 & -46.513 & 0.25 & 1149 & 58995 & 0.014876 & 271 & 6.5 \\
\hline
\end{tabular}

Table 2. Spectral properties of the different $\mathrm{TeV}$ extraction regions.

\begin{tabular}{lcccc}
\hline \hline Region & $\begin{array}{c}\Phi_{0}(1 \mathrm{TeV}) \\
10^{-12} \mathrm{TeV}^{-1} \mathrm{~cm}^{-2} \mathrm{~s}^{-1}\end{array}$ & $\Gamma$ & $\begin{array}{c}F(>0.2 \mathrm{TeV}) \\
10^{-11} \mathrm{~cm}^{-2} \mathrm{~s}^{-1}\end{array}$ & $\%$ total \\
\hline Full & $9.0 \pm 1.4$ & $2.19 \pm 0.08$ & $5.2 \pm 0.9$ & 100 \\
$A$ & $2.1 \pm 0.3$ & $2.11 \pm 0.12$ & $1.1 \pm 0.2$ & $21 \pm 4$ \\
$B$ & $1.4 \pm 0.2$ & $2.29 \pm 0.17$ & $0.8 \pm 0.2$ & $15 \pm 4$ \\
\hline
\end{tabular}

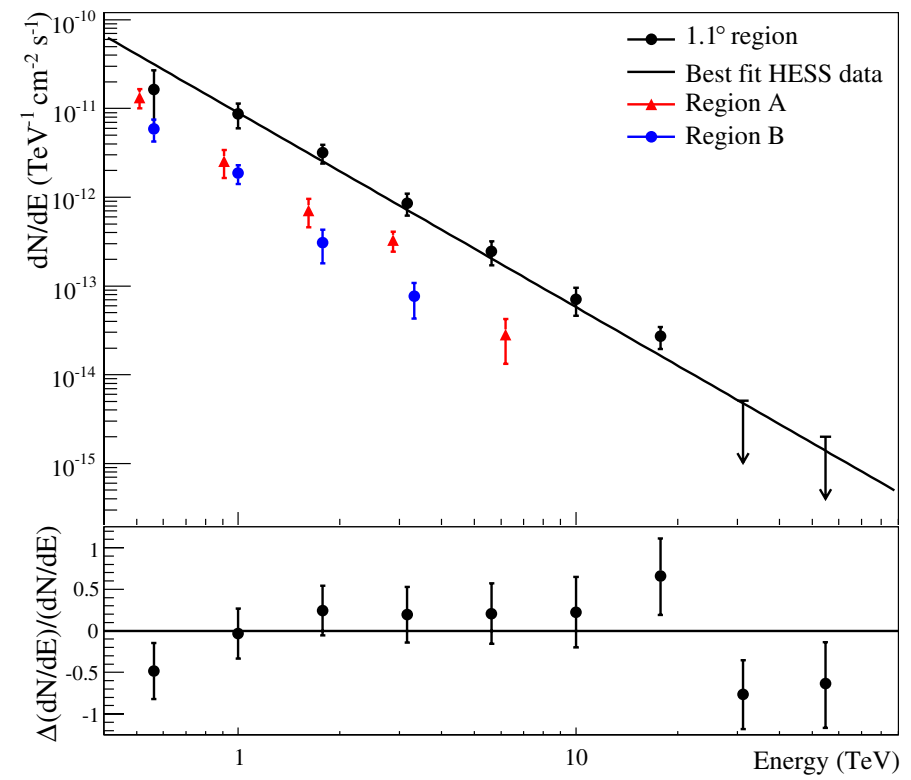

Fig. 4. Top: differential VHE $\gamma$-ray energy spectrum of HESS J1646-458. The data are fit by a power law $\mathrm{d} N / \mathrm{d} E=$ $\Phi_{0} \times(E / 1 \mathrm{TeV})^{-\Gamma}$. Arrows indicate the $95 \%$ upper limits for spectral bins which are compatible with a zero flux within $1 \sigma$. Also shown are spectra as obtained for region $A$ and $B$. Bottom: residuals of the power-law fit for the $1.1^{\circ} \gamma$-ray emission region.

spectra from region $A$ and $B$ does not allow to substantiate the preference for a two-source scenario further. Owing to this ambiguity, we pursue both possibilities through all portrayed counterpart scenarios.

Due to the large size of HESS J1646-458 $\left(\sim 2^{\circ}\right)$, a few objects that could be regarded as potential VHE $\gamma$-ray emitter are found in the region of interest. Besides the massive stellar cluster Westerlund 1, a high spin-down power pulsar PSR J1648-4611 (Manchester et al. 2005) which has recently been discovered to be a high-energy $\gamma$-ray pulsar with a possible unpulsed $\gamma$-ray component is found (Kerr 2009; Abdo et al. 2010, 2011). Furthermore, the low-mass X-ray binary (LMXB) 4U 1642-45 (Forman et al. 1978), the magnetar candidate CXOU J164710.2-455216 (Muno et al. 2006a) and three unidentified Fermi-LAT sources, 2FGL J1650.6-4603c, 2FGL J1651.8-4439c and 2FGL J1653.9-4627c (Abdo et al. 2011) are located within HESS J1646-458 (Fig. 5).

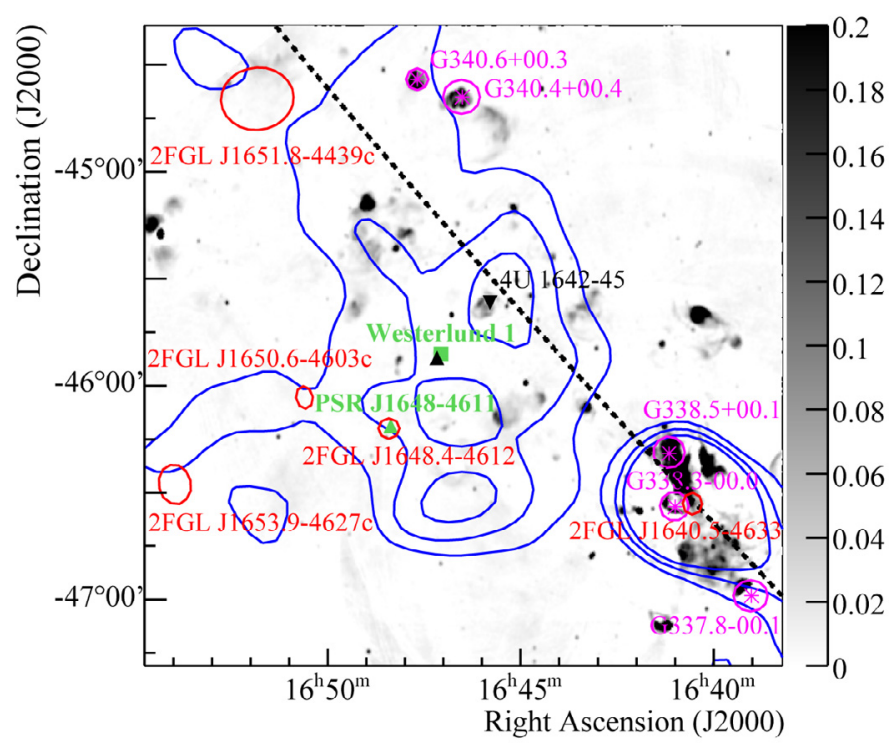

Fig. 5. H.E.S.S. smoothed VHE $\gamma$-ray excess contours in blue overlaid on the Molonglo $843 \mathrm{MHz}$ map (grey scale, in Jy/beam; Green et al. 1999). Also shown are SNRs (purple, Green 2009), the pulsar PSR J1648-4611 (Manchester et al. 2005), the LMXB 4U 1642-45 (Forman et al. 1978), Fermi-LAT sources (Abdo et al. 2011), the magnetar CXOU J164710.2-455216 (Muno et al. 2006a) as a black upright triangle and the stellar cluster $\mathrm{Wd} 1$.

In the following, the discussion will focus only on the astrophysically associated objects with known distance and energetics.

\section{1. $4 \cup 1642-45$}

The X-ray bright LMXB 4U 1642-45 with an accreting neutron star lies within the strong emission region $A$ (Fig. 5) and is located at a distance of 8.5 to $11.8 \mathrm{kpc}$ (van Paradijs \& White 1995; Grimm et al. 2002; Gilfanov et al. 2003). It exhibits an average X-ray luminosity of $L_{\mathrm{X}} \approx 10^{38} \mathrm{erg} \mathrm{s}^{-1}$ and Grimm et al. (2002) reported of periods of super-Eddington luminosity from ASM observations.

So far, only high-mass X-ray binary systems have been associated with point-like and variable VHE $\gamma$-ray sources (e.g. LS 5039, Aharonian et al. 2005, 2006e). At 8.5 to $11.8 \mathrm{kpc}$, HESS J1646-458 would be 320 to 450 pc in size and region 
H.E.S.S. Collaboration: VHE $\gamma$-ray emission from the vicinity of Westerlund 1

Table 3. Properties and inferred $\gamma$-ray luminosities of the pulsars and the VHE $\gamma$-ray emission regions.

\begin{tabular}{lcccccc}
\hline \hline Object / Region & $\begin{array}{c}\tau \\
10^{5} \mathrm{yrs}\end{array}$ & $\begin{array}{c}d \\
\mathrm{kpc}\end{array}$ & $\begin{array}{c}P_{0} \\
\mathrm{~s}\end{array}$ & $\begin{array}{c}\dot{P} \\
10^{-13} \mathrm{~s} \mathrm{~s}^{-1}\end{array}$ & $\begin{array}{c}\dot{E} \\
10^{34} \mathrm{erg} \mathrm{s}^{-1}\end{array}$ & $\begin{array}{c}L_{\gamma}{ }^{(1)} \\
10^{34} \mathrm{erg} \mathrm{s}^{-1}\end{array}$ \\
\hline CXOU J164710.2-455216 & 1.8 & 5 & 10.6 & 9.2 & $3 \times 10^{-3}$ & 26 \\
PSR J1648-4611 & 1.1 & 5.7 & 0.2 & 0.2 & 21 & 34 \\
\hline HESS J1646-458 & - & 4.3 & - & - & - & 19 \\
Region $A$ & - & 4.3 & - & - & - & 4.6 \\
Region $B$ & - & 4.3 & - & - & - & 2.9 \\
\hline
\end{tabular}

Notes. ${ }^{(1)}$ Obtained by scaling the observed VHE $\gamma$-ray flux between 0.1 and $100 \mathrm{TeV}$ to the nominal or adopted distance of the respective object. References. ${ }^{(a)}$ Muno et al. (2006a); Israel et al. (2007). ${ }^{(b)}$ Manchester et al. (2005).

A 100 to $144 \mathrm{pc}$, for angular diameters of $2.2^{\circ}$ and $0.7^{\circ}$ respectively. These inferred source sizes are well beyond the H.E.S.S. PSF in this analysis ( 26 to $35 \mathrm{pc}$ in diameter). A check for variability at the position of 4U 1642-45 did not reveal any indication of such as has been observed, e.g. in the case of LS 5039 and HESS J0632+057 (Aharonian et al. 2006e, 2007).

In summary, an association of 4U 1642-45 and the entire VHE $\gamma$-ray emission region is unlikely. The extent of the subregion $A$ also disfavours a scenario with 4U 1642-45 and a subregion as well. An association with region $A$ would be solely based on spatial coincidence but would then present a new class of VHE $\gamma$-ray sources.

\subsection{CXOU J164710.2-455216}

This anomalous X-ray pulsar is considered a magnetar and has been associated with $\mathrm{Wd} 1$ given its apparent proximity to the cluster and the low probability of a random association (Muno et al. 2006a). Shortly after its X-ray discovery, an outburst in X-rays was reported from Swift observations (Campana \& Israel 2006). Additionally, XMM-Newton observations were conducted prior to and after the outburst (Muno et al. 2007; Israel et al. 2007).

The observed X-ray luminosity is $L_{\mathrm{X}} \approx 3 \times 10^{33} \mathrm{erg} \mathrm{s}^{-1}$ which increased during the outburst by a factor of $\sim 100$. The rotation period is $10.6 \mathrm{~s}$ with a derivative of $\dot{P}=9.2 \times 10^{-13} \mathrm{~s} \mathrm{~s}^{-1}$ which infers an X-ray spin-down power of $\dot{E}=3 \times 10^{31} \mathrm{erg} \mathrm{s}^{-1}$ and a characteristic age of $\tau=1.8 \times 10^{5} \mathrm{yrs}$. The surface magnetic-field strength is $\sim 1 \times 10^{14} \mathrm{G}$. The relevant properties of CXOU J164710.2-455216 are listed in Table 3.

Since the X-ray luminosity exceeds the rotational spin-down power, this suggests that the observed X-ray emission is not due to spin-down processes, but some other form. For magnetars, the intense magnetic field can yield considerable power in magnetic dissipative processes. Following Zhang (2003), such power can be of the order $L_{\mathrm{mag}} \approx 10^{35} \mathrm{erg} \mathrm{s}^{-1}$, which, for CXOU J164710.2-455216, would dominate over the rotational spin-down power. If acceleration of particles to $\mathrm{TeV}$ energies occurs due to magnetic energy release one could expect a rather compact $\mathrm{TeV}$-emission region similar to spin-down powered $\mathrm{TeV}$ pulsar wind nebulae (PWNe) like the Crab nebula (Aharonian et al. 2006a).

However, it is still under investigation if magnetars can exhibit PWNe in general. For most pulsars, the respective PWNe would be too faint to be detected in X-rays (Gaensler $\&$ Slane 2006). In the VHE $\gamma$-ray regime, young $\left(O\left(10^{3} \mathrm{yrs}\right)\right)$ and high spin-down power $\left(O\left(10^{34} \mathrm{erg} \mathrm{s}^{-1}\right)\right)$ magnetars seem to be promising objects (Halpern \& Gotthelf 2010).

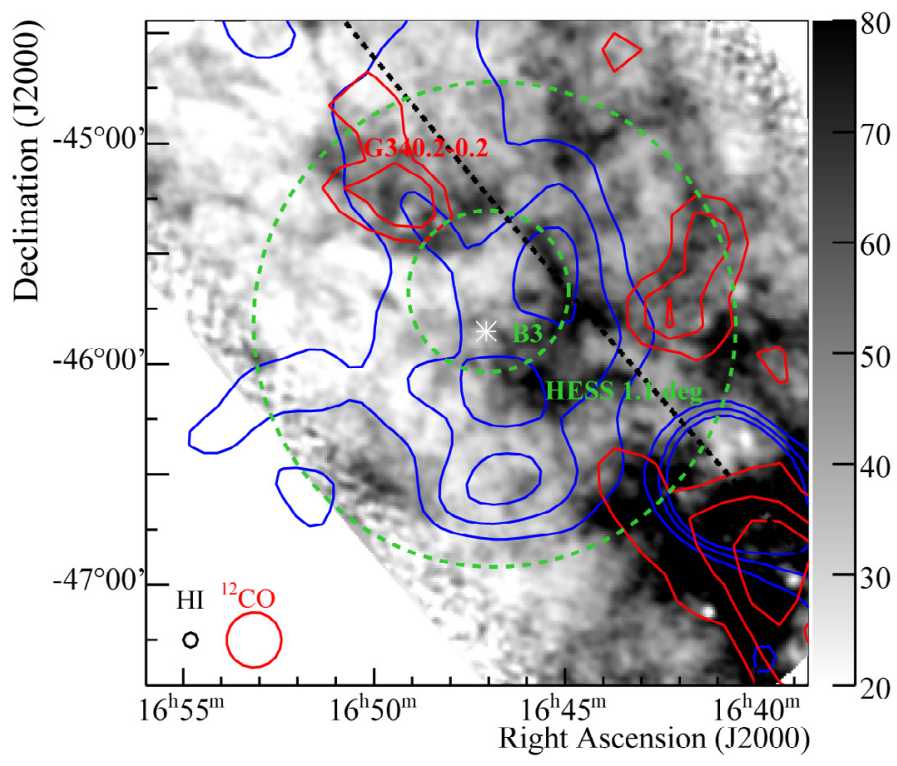

Fig. 6. HI $21 \mathrm{~cm}$ line emission map at a radial velocity of about $-55 \mathrm{~km} \mathrm{~s}^{-1}$ (McClure-Griffiths et al. 2005) between 20 and $80 \mathrm{~K}$. Bright regions are HI-depleted whereas dark regions are HI-dense. Overlaid are the CO contours (red) at 12.5, 22.5 and $32.5 \mathrm{~K}$ (Dame et al. 2001) along with the smoothed HESS J1646-458 contours in blue. The estimated position of the HI void $B 3$ is marked by the small green dashed circle (Kothes \& Dougherty 2007). The large green dashed circle indicates the region used for the spectral reconstruction in the $\mathrm{TeV}$ regime and to compute the ambient matter density. The position of $\mathrm{Wd} 1$ is marked by a white star in the centre. The circles in the lower left corner indicate the beam sizes of the respective radio observations.

However, the rotational spin-down power of CXOU J164710.2-455216 is too low to account for both the entire observed VHE $\gamma$-ray emission and either subregion. Therefore, any acceleration of particles would have to involve a magnetic energy release with a power output of $L_{\mathrm{mag}} \geq$ $10^{35} \mathrm{erg} \mathrm{s}^{-1}$. The observed emission could then be explained by an energy conversion process that operates with efficiency up to $100 \%$ but the size of HESS J1646-458 ( 160 pc at the distance of the magnetar) stands in contradiction to the expected compact region. Either of the two subregions could be energetically explained with efficiencies in the order of 0.5 but again as one would expect the resulting PWN to be compact and close to the magnetar itself, these scenarios are disfavoured as well.

All in all, VHE $\gamma$ rays from CXOU J164710.2-455216 is not a favoured scenario to account for HESS J1646-458 or parts from it as its relevant properties and current (V)HE $\gamma$-ray observations do not support such an approach. 


\subsection{PSR J1648-4611}

Among known VHE $\gamma$-ray sources, PWNe are the most abundant source type: Roughly one third of these sources are associated with PWN systems (e.g. Hinton \& Hofmann 2009). The pulsar is found to be located in the centre of the nebula (e.g. the Crab nebula, Aharonian et al. 2006a) or offset from it (e.g. the Vela X nebula, Aharonian et al. 2006b). Most of the VHE $\gamma$-ray-emitting PWNe are spatially extended and offset from the pulsar position and efficient in terms of converting available spin-down power into $\gamma$-ray emission. In general, PWNe firmly associated with known pulsars convert $10 \%$ at most of their spin-down power into $\gamma$-ray luminosity (Gallant 2007).

The high spin-down power pulsar PSR J1648-4611 with $\dot{E}=2.1 \times 10^{35} \mathrm{erg} \mathrm{s}^{-1}$ (Manchester et al. 2005) is located within the emission region HESS J1646-458 and associated with the Fermi-LAT source 2FGL J1648.4-4612 (Abdo et al. 2011). Pulsed $\gamma$-ray emission with the rotation period of PSR J1648-4611 as well as signatures for a constant (possible PWN) contribution have been reported. Therefore, this object has to be considered a $\gamma$-ray pulsar at low $\mathrm{GeV}$ energies. Inspection of archival Chandra data with $10 \mathrm{ks}$ exposure (obsid: 11836) shows no indication for the presence of an X-ray counterpart to PSR J1648-4611. Following the relation between spin-down power and X-ray luminosity of PWNe (Mattana et al. 2009), the expected X-ray luminosity $L_{X} \approx 5 \times 10^{30} \mathrm{erg} \mathrm{s}^{-1}$ of the associated PWN would not be detectable with current X-ray instruments and is hence consistent with the non-detection with Chandra.

The first attempt to explain HESS J1646-458 is a singlesource scenario which is motivated by the presence of the HE pulsar PSR J1648-4611. The inferred $\gamma$-ray luminosity $(0.1-100 \mathrm{TeV})$ at the distance of PSR J1648-4611 would require a conversion efficiency $\epsilon_{\mathrm{eff}} \approx 1$. Additionally, the size of the VHE $\gamma$-ray-emitting region would extend over roughly $200 \mathrm{pc}$ which is a factor 3-10 larger than known extended VHE $\gamma$-rayemitting PWN systems. Given the size, cooling losses would lead to considerable softening of the VHE $\gamma$-ray spectrum and a downward shift of the maximum energy with increasing distance from the pulsar - similar to the spectral softening of HESS J1825-137 (Aharonian et al. 2006d). The VHE $\gamma$-ray photon index reconstructed at the position of PSR J1648-4611 is $\Gamma=2.37 \pm 0.43$ using the reflected-background regions method and assuming a point-like source origin. This is compatible within statistical errors with the emission of the entire region. However, the available statistics do not permit firm conclusions about potential spectral changes across HESS J1646-458 to be drawn. In summary, the unprecedented high efficiency needed and the size of the VHE $\gamma$-ray emission region disfavour HESS J1646-458 as a very extended PWN.

In a two-source approach as motivated in Sect. 3.2, a displacement of PSR J1648-4611 from either subregion is apparent. The pulsar is displaced by $\sim 50-70 \mathrm{pc}$ from region $A$ and $B$. These offsets are large compared to those of known VHE $\gamma$-ray PWNe but could in principle be explained by relative proper motion of the pulsar assuming a transversal velocity of $O\left(500 \mathrm{~km} \mathrm{~s}^{-1}\right)$ which is at the upper end of the range of known transversal motions of pulsars (Manchester et al. 2005). In this scenario, one of the two subregions could in principle be powered by the pulsar with $\epsilon_{\mathrm{eff}} \approx 0.1 \ldots 0.2$. In this case, the morphology would reflect the ambient conditions, e.g. one of the subregions could be the result of the expansion of the PWN into a low-density medium or could be due to an asymmetric reverse shock of the SNR.
Recently, Luna et al. (2010) proposed that a region of low density in $\mathrm{CO}$ seems to partially match structures in preliminary VHE $\gamma$-ray data (Ohm et al. 2010b) and for which a SN event with PSR J1648-4611 as precursor could be responsible. However, the kinematic age of the cavity is about 55 times larger than the characteristic age of PSR J1648-4611 and the inferred subsonic expansion velocity is insufficient to accelerate particles up to the VHE $\gamma$-ray regime. Moreover, other SNR candidates at the position of this cavity are not to be found in archival data and the morphology of HESS J1646-458 does not strongly motivate a shell-like structure centred at about $16^{\mathrm{h}} 47^{\mathrm{m}} 23.3^{\mathrm{s}},-45^{\circ} 42^{\prime} 5.2^{\prime \prime}$ with an inner radius of $\sim 0.5^{\circ}$ (Luna et al. 2010). Although it cannot be ruled out that this cavity could be blown as a consequence of a SN it seems unplausible that the whole $\sim 25$ pc-thick shell would expand uniformly at $6-8 \mathrm{~km} \mathrm{~s}^{-1}$ and thereby giving rise to particle acceleration up to $\mathrm{TeV}$ energies.

Similarly as concluded for the magnetar, HESS J1646-458 seems unlikely to be explained as a very extended PWN powered by PSR J1648-4611. Either one of the subregions could be explained by an offset PWN. The inferred offsets would be comparatively large but the required efficiency would be amongst known TeV PWNe.

\subsection{Westerlund 1}

The motivation for H.E.S.S. observations of the $\mathrm{Wd} 1$ region has been outlined earlier (see Sect. 1) and the analysis of HESS J1646-458 revealed that Wd 1 is located close to the centroid of the VHE $\gamma$-ray emission. Hence this massive cluster is an interesting object to consider. As some of its properties are still subject of on-going investigation certain assumptions are made: (1) Due to the high visual extinction of $A_{v} \approx 12^{\mathrm{mag}}$, distance estimates based on photometry and spectroscopy vary strongly between $1.1 \mathrm{kpc}$ and $5.5 \mathrm{kpc}$ (Westerlund 1987; Piatti et al. 1998; Clark et al. 2005; Brandner et al. 2008). Kothes \& Dougherty (2007) derived a distance of $3.9 \mathrm{kpc}$ based on the HI line emission which Luna et al. (2010) extrapolated to $4.3 \mathrm{kpc}$ using the IAU distance recommendation to the Galactic Centre of $8.5 \mathrm{kpc}$. In this work, we adopt the $4.3 \mathrm{kpc}$ albeit using a more recent Galactocentric distance estimate of $8.33 \mathrm{kpc}$ (Gillessen et al. 2009). (2) Different model-dependent approaches to estimate the age suffer from the apparent lack of main-sequence stars. Recently, Negueruela et al. (2010) estimated the age to be $\gtrsim 5 \mathrm{Myr}$ and an age of $5 \mathrm{Myr}$ is adopted here. (3) A cluster mass of $10^{5} M_{\odot}$ is assumed.

Estimates based on the stellar population of $\mathrm{Wd} 1$ imply that 80 to 150 stars with initial masses exceeding $50 M_{\odot}$ have already undergone a SN explosion (Muno et al. 2006b). However, neither radio nor X-ray observations have revealed any SNR candidate. In order to estimate the total kinetic energy dissipated by the system through SNe and stellar winds the Starburst99 cluster evolution model (Leitherer et al. 1999; Vázquez \& Leitherer 2005; Leitherer et al. 2010) has been used. The default parameters with the standard Kroupa initial mass function (with the exponents 1.3 and 2.3, (Kroupa 2002)) and the evolutionary-model option, have been chosen. The total energy dissipated at the nominal age of $\mathrm{Wd} 1$ including stellar winds and $\mathrm{SNe}$ amounts to $E_{\text {kin }}=3.0 \times 10^{53} \mathrm{erg}$.

For an adiabatically expanding wind (Weaver et al. 1977; Silich et al. 2005), a structured and expanding $\left(\sim 30 \mathrm{~km} \mathrm{~s}^{-1}\right)$ hot bubble with an outer shock radius of $\sim 250 \mathrm{pc}$ is expected to form around Wd 1 even if only a moderate number of $50 \mathrm{OB}$ stars are considered. However, a dedicated search for such a super bubble-like structure in $\mathrm{HI}$ and $\mathrm{CO}$ data at the radial velocity of 
Wd 1 did not reveal any signatures of such a feature (McClureGriffiths et al. 2005; Kothes \& Dougherty 2007). The latter authors find indications for a much smaller $(55 \mathrm{pc})$ and slowly expanding $\left(3 \mathrm{~km} \mathrm{~s}^{-1}\right)$ feature (B3 in Kothes \& Dougherty 2007), see Fig. 6. However, in this first approximation, radiative cooling is not considered although this cooling process might affect the evolution of stellar cluster winds (e.g. discussed in Wünsch et al. 2008).

Kothes \& Dougherty (2007) interpret the formation of the HII region complex G340.2-0.2 (depicted in Fig. 6) as triggered by the stellar wind activity of $\mathrm{Wd} 1$. Indeed, if $\mathrm{Wd} 1$ is located at a distance of $4.3 \mathrm{kpc}$ some correlation between VHE $\gamma$ rays and the location of this HII region is expected.

An average gas density for atomic hydrogen $n_{\mathrm{HI}}$ and molecular gas $n_{\mathrm{H}_{2}}$ can be derived using available $\mathrm{HI}$ and $\mathrm{CO}$ data in the velocity range of $\mathrm{Wd} 1\left(-50\right.$ to $\left.-60 \mathrm{~km} \mathrm{~s}^{-1}\right)$ for the entire $1.1^{\circ}$ region of interest. For this, all pixel values in this velocity range and within $1.1^{\circ}$ from the cluster position are considered as well as the SGPS beam size (130 arcsecs) and the oversampling factor (11.97). With the HI intensity-mass conversion factor of $1.823 \times 10^{18} \mathrm{~cm}^{-2} /\left(\mathrm{K} \mathrm{km} \mathrm{s}^{-1}\right)$ (Yamamoto et al. 2003), the result $^{2}$ is $n_{\mathrm{HI}}=0.24 \mathrm{~cm}^{-3}$. In the CO data (Dame et al. 2001), a low-density region around the cluster seems to be apparent as well (Fig. 6). This feature is larger than B3. With a beam size of 450 arcsecs and a oversampling factor of 1 , the application of the conversion factor of $\mathrm{CO}$ to $\mathrm{H}_{2}$ of $1.5 \times 10^{20} \mathrm{~cm}^{-2} /\left(\mathrm{K} \mathrm{km} \mathrm{s}^{-1}\right)$ (Strong et al. 2004) leads to an average density of $\mathrm{H}_{2}$ molecules in units of atomic hydrogen of $n_{\mathrm{H}_{2}}=12.16 \mathrm{~cm}^{-3}$. Note that the $\mathrm{X}$ factor used for the $\mathrm{CO}$ data incorporates caveats pointed out in Strong et al. (2004). The required energy in CRs to power the $\gamma$-ray emission can now be estimated:

$E_{\mathrm{CR}}=2.1 \times 10^{50}\left(\frac{L_{>1 \mathrm{GeV}}}{5.8 \times 10^{35} \mathrm{erg} \mathrm{s}^{-1}}\right)\left(\frac{n_{\mathrm{HI}}+n_{\mathrm{H}_{2}}}{12.4 \mathrm{~cm}^{-3}}\right)^{-1} \mathrm{erg}$,

where $L_{>1 \mathrm{GeV}}$ is the high-energy luminosity computed with the spectral results presented earlier between $1 \mathrm{GeV}$ and $1 \mathrm{PeV}$.

When interpreted in a single-source scenario, the required efficiency for transferring kinetic energy in shocks or turbulences into energetic particles through acceleration is therefore at the level of $\sim 0.1 \%$. For a similar argument as for the PWN interpretation, a leptonic origin of the VHE $\gamma$-ray emission is difficult to reconcile with the large extent of HESS J1646-458 which translates into a size of $160 \mathrm{pc}$ at the distance of the cluster. Given the large photon density in the environment of $\mathrm{Wd} 1$, a fast convective transport of the electrons would be required to prevent them from cooling. Hence a dominant hadronic origin is favoured in this approach.

A possible two-source scenario would also consist of a dominant hadronic CR component as the stellar photon field would lead to a rapid cooling of VHE electrons. In this case, bright VHE $\gamma$-ray structures would trace dense features in $\mathrm{HI}$ and $\mathrm{CO}$. In particular, region $A$ lies at the edge of $B 3$ and is coincident with dense structures in HI (see Fig. 6) which would naturally provide sufficient target material. Region $B$, however, remains comparatively dark as $\mathrm{HI}$ and $\mathrm{CO}$ data do not suggest a higher abundance of target material.

In summary, Wd 1 and its massive stars favour a hadronic mechanism for the entire emission region. Here, the size and the

\footnotetext{
2 The density $n$ is proportional to $\sum b^{2} p_{i} R^{-1} f_{\mathrm{s}}^{-2}$ where $p_{i}$ are the pixel values of the HI or CO map, $R$ the radius of the region of interest, $b$ the beam size of the respective experiment and $f_{\mathrm{s}}$ is the oversampling factor in the respective HI or CO maps used.
}

inferred energetics of HESS J1646-458 could be plausibly explained. In case of the two subregions, or multiple source regions in general, more observational data in all wavelength bands and detailed modelling are required.

\section{Summary and conclusions}

In summary, HESS J1646-458 is a new VHE $\gamma$-ray source found towards the unique massive stellar cluster Westerlund 1 and a number of other potential counterparts. The large size of HESS J1646-458 however, over 2 degrees in diameter making it one of the largest $\mathrm{TeV}$ sources so far detected by H.E.S.S., presents a challenge in identifying a clear counterpart (or a number of counterparts) to explain the VHE $\gamma$-ray emission.

The detection of degree-scale VHE $\gamma$-ray emission, namely HESS J1646-458, towards the stellar cluster Westerlund 1 with a total significance of $20 \sigma$ from H.E.S.S. observations performed in the years 2004, 2007 and 2008 (33.8 hrs live time) is reported. The energy spectrum between $0.45 \mathrm{TeV}$ and $75 \mathrm{TeV}$ of the entire region is best fit by a simple power law with an index $\Gamma=2.19 \pm 0.08_{\text {stat }} \pm 0.2_{\text {sys }}$ and a normalisation at $1 \mathrm{TeV} \Phi_{0}=\left(9.0 \pm 1.4_{\text {stat }} \pm 1.8_{\text {sys }}\right) \times 10^{-12} \mathrm{TeV}^{-1} \mathrm{~cm}^{-2} \mathrm{~s}^{-1}$ with $\chi^{2} /$ ndf $=9.9 / 7$. The integrated flux above $0.2 \mathrm{TeV}$ amounts to $(3.49 \pm 0.52) \times 10^{-11} \mathrm{~cm}^{-2} \mathrm{~s}^{-1}$. The VHE $\gamma$-ray luminosity between 0.1 and $100 \mathrm{TeV}$ is $1.9 \times 10^{35}\left(d / 4.3 \mathrm{kpc}^{2} \mathrm{erg} \mathrm{s}^{-1}\right.$.

The centroid of HESS J1646-458 is consistent with the nominal position of $\mathrm{Wd} 1$. The observed VHE $\gamma$-ray emission region has a diameter of about $2^{\circ}$ which translates into a spatial extent of $160 \mathrm{pc}$ at the distance of $\mathrm{Wd} 1$ or $200 \mathrm{pc}$ at the distance of PSR J1648-4611. In either case, the size of HESS J1646-458 would be the largest among currently known VHE $\gamma$-ray sources, if the emission is of a single-source origin. This is supported by the lack of spectral changes across HESS J1646-458 within statistical errors. Although there is some evidence for a multisource morphology, the limited statistics hamper a detailed investigation into the presence of multiple sources.

In a scenario where one astrophysical object is responsible for HESS J1646-458, Wd 1 could naturally account for the required injection power provided that about $0.1 \%\left(n / 12.4 \mathrm{~cm}^{-3}\right)$ of the kinetic energy released by stellar winds and supernova explosions are converted into particle acceleration. In this case, the stellar wind and SNe activity of Wd 1 would strongly affect the surroundings but also reflect the ambient conditions.

In a split of HESS J1646-458 into two distinct subregions, however, a superposition of two or more sources adding up to the observed VHE $\gamma$-ray emission region could be possible. In this case, one of the subregions could also be explained by a PWN with comparatively large offset from PSR J1648-4611. Despite the spatial coincidence, the LMXB 4U 1642-45 and the magnetar CXOU 164710.2-455216 are not likely to account for HESS J1646-458 or parts of it.

Further multiwavelength observations (in particular those covering the VHE $\gamma$-ray source in full), and deeper VHE $\gamma$-ray coverage will no doubt be valuable in shedding light on the nature of this source.

Acknowledgements. The support of the Namibian authorities and of the University of Namibia in facilitating the construction and operation of H.E.S.S. is gratefully acknowledged, as is the support by the German Ministry for Education and Research (BMBF), the Max Planck Society, the French Ministry for Research, the CNRS-IN2P3 and the Astroparticle Interdisciplinary Programme of the CNRS, the UK Particle Physics and Astronomy Research Council (PPARC), the IPNP of the Charles University, the South African Department of Science and Technology and National Research Foundation, and by the University of Namibia. We appreciate the excellent work of the technical support staff in Berlin, Durham, Hamburg, Heidelberg, Palaiseau, Paris, 
Saclay, and in Namibia in the construction and operation of the equipment. This research has made use of NASA's Astrophysics Data System. This research has made use of the SIMBAD database, operated at CDS, Strasbourg, France. SO acknowledges the support of the Humboldt foundation by a Feodor-Lynen research fellowship.

\section{References}

Abdo, A. A., Ackermann, M., Ajello, M., et al. 2010, Fermi/Large Area Telescope 1 year catalog, http://fermi.gsfc.nasa.gov/ssc/data/ access/lat/1yr_catalog/1FGL_catalog_v2.pdf

Abdo, A. A., Ackermann, M., Ajello, M., et al. 2011, Fermi/Large Area Telescope 2 year catalog, http://fermi.gsfc.nasa.gov/ssc/data/ access/lat/2yr_catalog/

Abramowski, A., Acero, F., Aharonian, F., et al. 2011, A\&A, 525, A46

Acciari, V. A., Aliu, E., Arlen, T., et al. 2011, ApJ, 730, L20

Aharonian, F., Akhperjanian, A. G., Aye, K., et al. 2005, Science, 309, 746

Aharonian, F., Akhperjanian, A. G., Bazer-Bachi, A. R., et al. 2006b, A\&A, 448, L43

Aharonian, F., Akhperjanian, A. G., Bazer-Bachi, A. R., et al. 2006a, A\&A, 457, 899

Aharonian, F., Akhperjanian, A. G., Bazer-Bachi, A. R., et al. 2006c, ApJ, 636, 777

Aharonian, F., Akhperjanian, A. G., Bazer-Bachi, A. R., et al. 2006d, A\&A, 460, 365

Aharonian, F., Akhperjanian, A. G., Bazer-Bachi, A. R., et al. 2006e, A\&A, 460, 743

Aharonian, F. A., Akhperjanian, A. G., Bazer-Bachi, A. R., et al. 2007, A\&A, 469, L1

Axford, W. I., Leer, E., \& Skadron, G. 1977, in International Cosmic Ray Conference, 11, International Cosmic Ray Conference, 132

Bamba, A., Ueno, M., Nakajima, H., \& Koyama, K. 2004, ApJ, 602, 257

Bednarek, W. 2005, MNRAS, 363, L46

Bell, A. R. 1978, MNRAS, 182, 443

Benaglia, P., \& Romero, G. E. 2003, A\&A, 399, 1121

Berge, D., Funk, S., \& Hinton, J. 2007, A\&A, 466, 1219

Blandford, R. D., \& Ostriker, J. P. 1978, ApJ, 221, L29

Brandner, W., Clark, J. S., Stolte, A., et al. 2008, A\&A, 478, 137

Bykov, A. M. 2001, Space Sci. Rev., 99, 317

Campana, S., \& Israel, G. L. 2006, The Astronomer's Telegram, 893, 1

Casanova, S., Aharonian, F. A., Gabici, S., et al. 2009, in AIP Conf. Ser.

Chaves, R. C. G., de Oña Wilhemi, E., \& Hoppe, S. 2008, in AIP Conf. Ser. 1085, ed. F. A. Aharonian, W. Hofmann, \& amp, F. Rieger, 219

Clark, J. S., Negueruela, I., Crowther, P. A., \& Goodwin, S. P. 2005, A\&A, 434, 949

Clark, J. S., Negueruela, I., Ritchie, B., Crowther, P., \& Dougherty, S. 2010, The Messenger, 142, 31

Cooper, R. L., Guerrero, M. A., Chu, Y., Chen, C., \& Dunne, B. C. 2004, ApJ, 605,751

Crowther, P. A., Hadfield, L. J., Clark, J. S., Negueruela, I., \& Vacca, W. D. 2006, MNRAS, 372, 1407

Dame, T. M., Hartmann, D., \& Thaddeus, P. 2001, ApJ, 547, 792

de Naurois, M., \& Rolland, L. 2009, Astropart. Phys., 32, 231

Domingo-Santamaría, E., \& Torres, D. F. 2006, A\&A, 448, 613

Dougherty, S. M., Clark, J. S., Negueruela, I., Johnson, T., \& Chapman, J. M. 2010, A\&A, 511, A58

Eichler, D., \& Usov, V. 1993, ApJ, 402, 271

Ellison, D. C., Patnaude, D. J., Slane, P., \& Raymond, J. 2010, ApJ, 712, 287

Ferrand, G., \& Marcowith, A. 2010, A\&A, 510, A101

Forman, W., Jones, C., Cominsky, L., et al. 1978, ApJS, 38, 357

Gaensler, B. M., \& Slane, P. O. 2006, ARA\&A, 44, 17

Gallant, Y. A. 2007, Ap\&SS, 309, 197

Gies, D. R. 2008, in Massive Star Formation: Observations Confront Theory, ed.

H. Beuther, H. Linz, \& T. Henning, ASP Conf. Ser., 387, 93

Gilfanov, M., Revnivtsev, M., \& Molkov, S. 2003, A\&A, 410, 217

Gillessen, S., Eisenhauer, F., Trippe, S., et al. 2009, ApJ, 692, 1075

Green, D. A. 2009, Bull. Astron. Soc. India, 37, 45

Green, A. J., Cram, L. E., Large, M. I., \& Ye, T. 1999, ApJS, 122, 207

Grimm, H., Gilfanov, M., \& Sunyaev, R. 2002, A\&A, 391, 923

Halpern, J. P., \& Gotthelf, E. V. 2010, ApJ, 725, 1384

H.E.S.S. Collaboration, Abramowski, A., Acero, F., et al. 2011, 531, A81

Higdon, J. C., \& Lingenfelter, R. E. 2003, ApJ, 590, 822

Hillas, A. M. 1985, in International Cosmic Ray Conference, ed. F. C. Jones, 3, 445

Hinton, J. A., \& Hofmann, W. 2009, ARA\&A, 47, 523

Israel, G. L., Campana, S., Dall'Osso, S., et al. 2007, ApJ, 664, 448

Kerr, M. 2009
Kothes, R., \& Dougherty, S. M. 2007, A\&A, 468, 993

Kroupa, P. 2002, Science, 295, 82

Krymskii, G. F. 1977, Akademiia Nauk SSSR Doklady, 234, 1306

Law, C., \& Yusef-Zadeh, F. 2004, ApJ, 611, 858

Leitherer, C., Schaerer, D., Goldader, J. D., et al. 1999, ApJS, 123, 3

Leitherer, C., Ortiz Otálvaro, P. A., Bresolin, F., et al. 2010, ApJS, 189, 309

Luna, A., Mayya, Y. D., Carrasco, L., \& Bronfman, L. 2010, ApJ, 713, L45

Manchester, R. N., Hobbs, G. B., Teoh, A., \& Hobbs, M. 2005, AJ, 129, 1993

Manolakou, K., Horns, D., \& Kirk, J. G. 2007, A\&A, 474, 689

Mattana, F., Falanga, M., Götz, D., et al. 2009, ApJ, 694, 12

McClure-Griffiths, N. M., Dickey, J. M., Gaensler, B. M., et al. 2005, ApJS, 158, 178

Montmerle, T. 1979, ApJ, 231, 95

Mücke, A., \& Pohl, M. 2002, in Interacting Winds from Massive Stars, ed. A. F. J. Moffat \& N. St-Louis, ASP Conf. Ser., 260, 355

Muno, M. P., Clark, J. S., Crowther, P. A., et al. 2006a, ApJ, 636, L41

Muno, M. P., Law, C., Clark, J. S., et al. 2006b, ApJ, 650, 203

Muno, M. P., Gaensler, B. M., Clark, J. S., et al. 2007, MNRAS, 378, L44

Negueruela, I., Clark, J. S., \& Ritchie, B. W. 2010, A\&A, 516, A78

Ohm, S., van Eldik, C., \& Egberts, K. 2009, Astropart. Phys., 31, 383

Ohm, S., Fernandes, M. V., de Ona Wilhelmi, E., et al. 2010a, in 25th Texas Symposium on Relativistic Astrophysics

Ohm, S., Horns, D., Reimer, O., et al. 2010b, in ASP Conf. Ser. 422, ed. J. Martí, P. L. Luque-Escamilla, \& J. A. Combi, 265

Oskinova, L. M. 2005, MNRAS, 361, 679

Piatti, A. E., Bica, E., \& Claria, J. J. 1998, A\&AS, 127, 423

Reimer, A., Pohl, M., \& Reimer, O. 2006, ApJ, 644, 1118

Rowell, G. P. 2003, A\&A, 410, 389

Silich, S., Tenorio-Tagle, G., \& Añorve-Zeferino, G. A. 2005, ApJ, 635, 1116

Strong, A. W., Moskalenko, I. V., Reimer, O., Digel, S., \& Diehl, R. 2004, A\&A, 422, L47

van Paradijs, J., \& White, N. 1995, ApJ, 447, L33

Vázquez, G. A., \& Leitherer, C. 2005, ApJ, 621, 695

Weaver, R., McCray, R., Castor, J., Shapiro, P., \& Moore, R. 1977, ApJ, 218, 377

Westerlund, B. 1961, AJ, 66, 57

Westerlund, B. E. 1987, A\&AS, 70, 311

Wolk, S. J., Bourke, T. L., Smith, R. K., Spitzbart, B., \& Alves, J. 2002, ApJ, 580, L161

Wünsch, R., Tenorio-Tagle, G., Palouš, J., \& Silich, S. 2008, ApJ, 683, 683

Yamamoto, H., Onishi, T., Mizuno, A., \& Fukui, Y. 2003, ApJ, 592, 217

Zhang, B. 2003, in Proceedings of the International Workshop on Strong Magnetic Fields and Neutron Stars, 83

Zinnecker, H. 2003, in A Massive Star Odyssey: From Main Sequence to Supernova, ed. K. van der Hucht, A. Herrero, \& C. Esteban, IAU Symp., 212,80

Zinnecker, H., \& Yorke, H. W. 2007, ARA\&A, 45, 481

1 Universität Hamburg, Institut für Experimentalphysik, Luruper Chaussee 149, 22761 Hamburg, Germany

e-mail: milton.virgilio.fernandes@physik.uni-hamburg.de

${ }^{2}$ Laboratoire Univers et Particules de Montpellier, Université Montpellier 2, CNRS/IN2P3, CC 72, Place Eugène Bataillon, 34095 Montpellier Cedex 5, France

3 Max-Planck-Institut für Kernphysik, PO Box 103980, 69029 Heidelberg, Germany

4 Dublin Institute for Advanced Studies, 31 Fitzwilliam Place, Dublin 2, Ireland

5 National Academy of Sciences of the Republic of Armenia, Yerevan

6 Yerevan Physics Institute, 2 Alikhanian Brothers St., 375036 Yerevan, Armenia

7 Universität Erlangen-Nürnberg, Physikalisches Institut, ErwinRommel-Str. 1, 91058 Erlangen, Germany

${ }^{8}$ Nicolaus Copernicus Astronomical Center, ul. Bartycka 18, 00-716 Warsaw, Poland

9 CEA Saclay, DSM/IRFU, 91191 Gif-Sur-Yvette Cedex, France

10 University of Durham, Department of Physics, South Road, Durham DH1 3LE, UK

11 Astroparticule et Cosmologie (APC), CNRS, Université Paris 7 Denis Diderot, 10 rue Alice Domon et Léonie Duquet, 75205 Paris Cedex 13, France (UMR 7164: CNRS, Université Paris VII, CEA, Observatoire de Paris) 
12 Laboratoire Leprince-Ringuet, École Polytechnique, CNRS/IN2P3, 91128 Palaiseau, France

13 Institut für Theoretische Physik, Lehrstuhl IV: Weltraum und Astrophysik, Ruhr-Universität Bochum, D 44780 Bochum, Germany

14 Landessternwarte, Universität Heidelberg, Königstuhl, 69117 Heidelberg, Germany

15 Institut für Physik, Humboldt-Universität zu Berlin, Newtonstr. 15, 12489 Berlin, Germany

${ }^{16}$ LUTH, Observatoire de Paris, CNRS, Université Paris Diderot, 5 place Jules Janssen, 92190 Meudon, France

17 LPNHE, Université Pierre et Marie Curie Paris 6, Université Denis Diderot Paris 7, CNRS/IN2P3, 4 place Jussieu, 75252 Paris Cedex 5, France

18 Institut für Astronomie und Astrophysik, Universität Tübingen, Sand 1, 72076 Tübingen, Germany

19 Astronomical Observatory, The University of Warsaw, Al. Ujazdowskie 4, 00-478 Warsaw, Poland

20 Unit for Space Physics, North-West University, Potchefstroom 2520, South Africa

21 Laboratoire d'Annecy-le-Vieux de Physique des Particules, Université de Savoie, CNRS/IN2P3, 74941 Annecy-le-Vieux, France

22 Oskar Klein Centre, Department of Physics, Stockholm University, Albanova University Center, 10691 Stockholm, Sweden
23 University of Namibia, Department of Physics, Private Bag 13301, Windhoek, Namibia

${ }^{24}$ Laboratoire d'Astrophysique de Grenoble, INSU/CNRS, Université Joseph Fourier, BP 53, 38041 Grenoble Cedex 9, France

25 Department of Physics and Astronomy, The University of Leicester, University Road, Leicester, LE1 7RH, UK

e-mail: physoh@leeds.ac.uk

26 Instytut Fizyki Jạdrowej PAN, ul. Radzikowskiego 152, 31-342 Kraków, Poland

27 Institut für Astro- und Teilchenphysik, Leopold-FranzensUniversität Innsbruck, 6020 Innsbruck, Austria

28 Obserwatorium Astronomiczne, Uniwersytet Jagielloński, ul. Orla 171, 30-244 Kraków, Poland

29 Toruń Centre for Astronomy, Nicolaus Copernicus University, ul. Gagarina 11, 87-100 Toruń, Poland

30 School of Chemistry \& Physics, University of Adelaide, Adelaide 5005, Australia

31 Charles University, Faculty of Mathematics and Physics, Institute of Particle and Nuclear Physics, V Holešovičkách 2, 18000 Prague 8, Czech Republic

32 School of Physics \& Astronomy, University of Leeds, Leeds LS2 9JT, UK

33 European Associated Laboratory for Gamma-Ray Astronomy, jointly supported by CNRS and MPG 